\title{
A Rare Case of Isolated Trichobezoar in Jejunum Presenting as Obstruction - A Case Report
}

\author{
Jujaray Deepti, Gontumukkala Chalapathi*, Vasu Burli, Malikireddy Hima Bindu and Chettipally Varuna Sree \\ Department of Pediatrics, KIMS Hospital, India
}

Submission: June 21, 2019; Published: July 05, 2019

*Corresponding author: Gontumukkala Chalapathi, Department of Pediatrics, KIMS Hospital, Kurnool, Andhra Pradesh, India

\begin{abstract}
Introduction: A trichobezoar is a rare condition that starts with ingestion of hair which gets accumulated and forms a mass that causes obstruction. This is most commonly seen in girls and stomach being the most common site. Rarely, seen as isolated mass in jejunum or the stomach mass may extend to jejunum which is then referred as Rapunzel syndrome.

Case report: This is a case of an 8yr old girl who presented with vomiting's and pain abdomen consistent with intestinal obstruction. No history of trichotillomania or trichophagia. Exploratory Laparotomy and Enterotomy revealed a $7 \times 5 \mathrm{~cm}$ mass in jejunum with no gastric component.

Conclusion: Trichobezoar is a rare surgically treatable condition. They should be considered in girls presenting with symptoms of obstruction. Parental counseling and psychotherapy should be mandatory in follow-up which help in preventing relapses. With high level of suspicion, prompt diagnosis and treatment, trichobezoars have a good prognosis.
\end{abstract}

Keywords: Trichobezoar; Trichophagia; Intestinal obstruction; Laparoscopy

\section{Introduction}

A trichobezoar is a mass formed by ingestion and accumulation of undigested hair inside the gastrointestinal tract. Stomach is the most common site which may extend to duodenum as well as jejunum, which is then referred as Rapunzel Syndrome. Incidence of trichobezoar in paediatric population is around $0.4 \%$ [1]. It is seen typically in girls usually with a history of trichotillomania and trichophagia. Affected children may remain unnoticed until it causes obstructive symptoms. We hereby report a unique case of isolated jejunal trichobezoar presenting as intestinal obstruction in an 8yr old girl.

\section{Case Presentation}

An 8yr old girl presented with complaints of pain abdomen for 6 days, vomiting's for 6 days initially non bilious and bilious for 3 days and not passed stools for 4 days. History of anorexia and weight loss was present. The child denied history of trichophagia but had patchy alopecia 2 years before this presentation for which she was treated. On examination, child was malnourished with moderate dehydration and pallor. Abdomen was tender all over with no palpable mass. Laboratory investigations showed anaemia (Hb-7\%), with marked leukocytosis (TLC - 21000cells/ cu $\mathrm{mm}$ ). USG revealed prominent fluid filled jejunal loops. CECT abdomen was suggestive of Subacute intestinal obstruction with dilated fluid filled ileal and jejunal loops with minimal ascites.
Child was subsequently taken for exploratory laparotomy which revealed an intraluminal mass in mid jejunum with dilated proximal jejunum (Figure 1). Enterotomy was done with vertical incision over the mass (Figure 2) and a trichobezoar of $7 \times 5 \mathrm{~cm}$ was removed (Figure 3). Child recovered well with an uneventful postoperative period. Child was discharged on $8^{\text {th }}$ postoperative day with psychiatric referral.

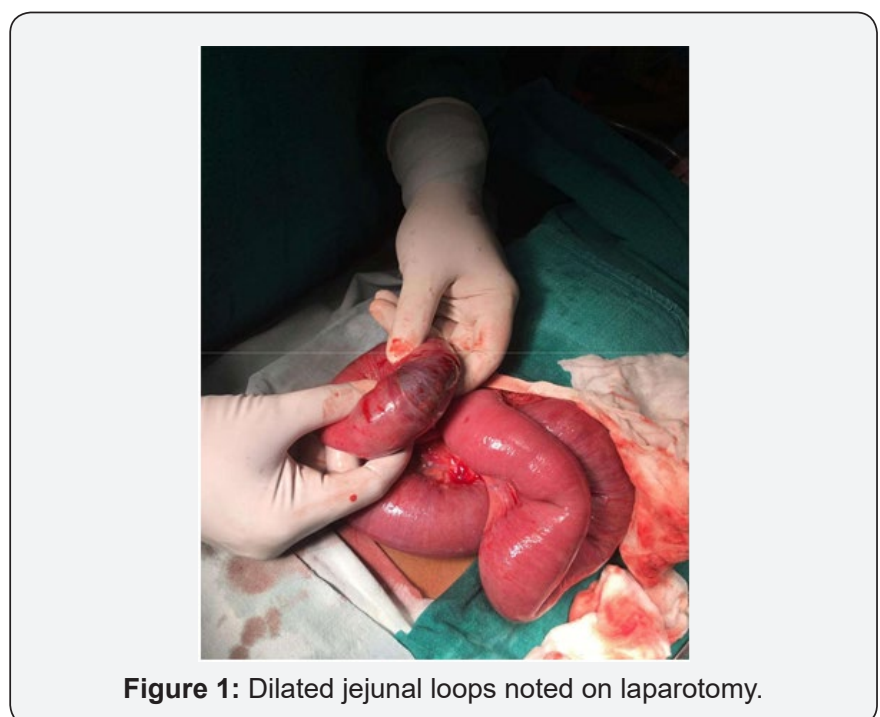




\section{Juniper Online Journal of Case Studies}
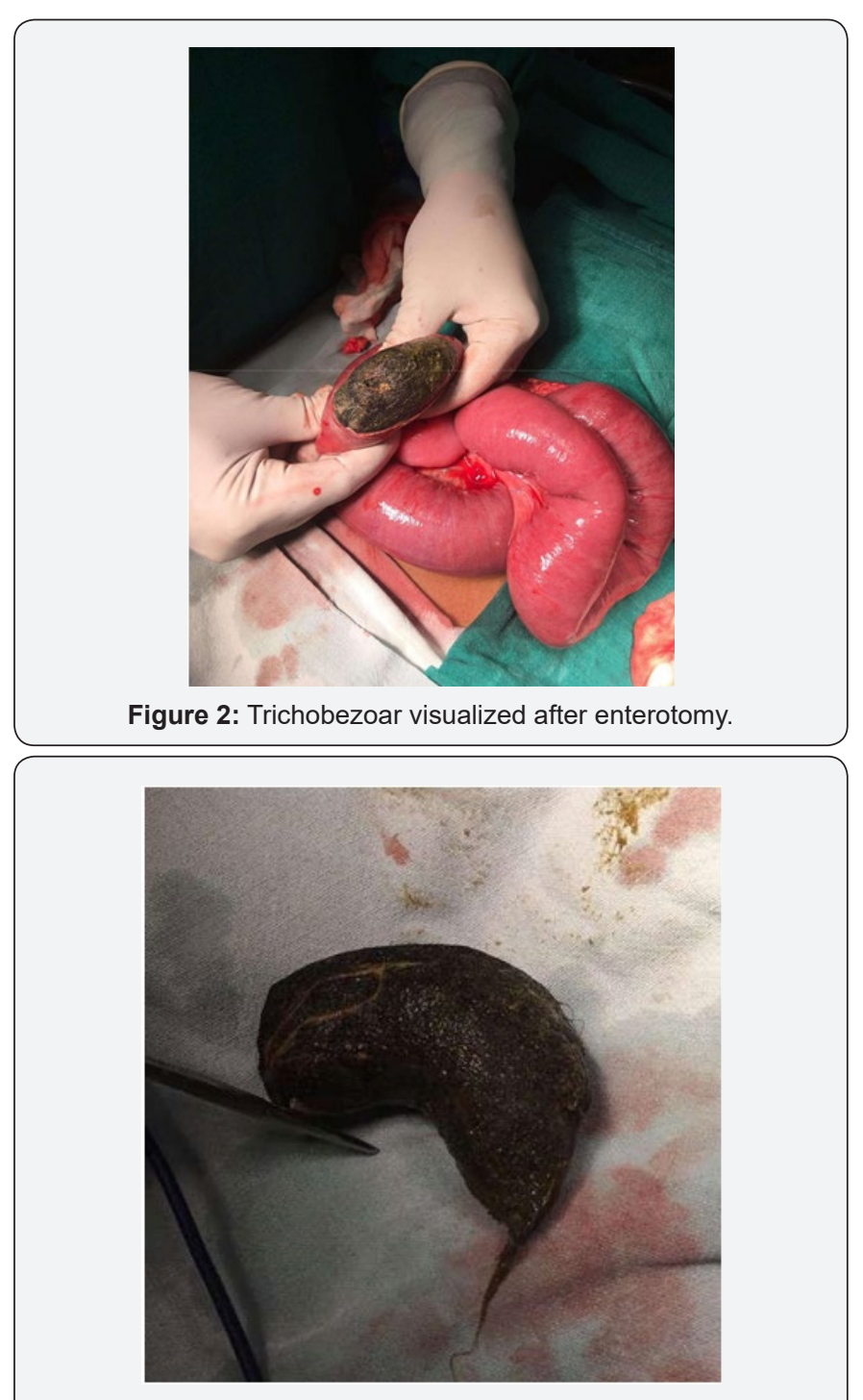

Figure 3: Specimen of trichobezoar removed from Jejunum.

Discussion

Trichobezoars are concretions of hair, a rare entity, usually seen in girls, stomach being the most common site. It can be seen in as young as 3 years old [2]. Trichobezoar occurs in 1\% of the patients with trichophagia [1]. However, they may appear even years after stopping trichophagia [3]. Psychiatric illness and mental retardation may be seen in about $10 \%$ of the children [4]. Smooth surface of hair makes it resistant to propulsion by peristalsis and gets retained slowly developing into a mass [5]. Gastric bezoars tear into pieces and get impacted in small intestine leading to obstruction. Extension from stomach to intestines is referred to as Rapunzel Syndrome. The gastric acid causes denaturation of hair changing its colour to black irrespective of their original colour [6]. A positive family history for psychiatric illnesses and consuming non nutritional substances may be seen in some children.

Recently, the incidence has been increasing with varied presentations. Trichobezoars may go undiagnosed until the size increases sufficiently to cause symptoms of obstruction. It may also present as volvulus or intussusception [7,8]. Clinically, 70\% of the children present with chronic or recurrent pain abdomen, nausea and vomiting is seen in $65 \%, 38 \%$ have anorexia and weight loss, altered gastrointestinal motility manifesting as diarrhoea or constipation in $33 \%$ of the children [6]. A welldefined abdominal mass is generally present which is mobile in $90 \%$ of the cases and may indent (Lamerton's sign) [9]. If left untreated trichobezoars grow and undergo complications including mucosal erosion, ulceration and perforation. These are the result of reduction in blood supply to mucosa of stomach and intestine. They may also lead to intestinal obstruction, volvulus, biliary perforation, intussusception in few children.

A high suspicion is required to diagnose trichobezoar preoperatively due to nonspecific presentation. A positive history of trichophagia along with radiological imaging plays an important role in diagnosis. Ultrasound abdomen is used as initial modality in diagnosing which shows a pathognomonic shadow between bezoar and bowel wall. CECT is the preferred modality, a heterogeneous mass with mottled gas pattern suggests a bezoar [10]. It helps in delineating the size and extension. Upper GI Endoscopy is the gold standard for gastric bezoars.

Management is mainly surgical intervention and the approach depends on the size and location. Laparotomy or laparoscopy is the best treatment modality. Small bezoars can be retrieved by laparoscopy but large ones and those with complications require open procedure as they are difficult to remove. In our case, trichobezoar was diagnosed intra operatively. Laparoscopy was preferred in our case due to its location in jejunum and a decent size of bezoar. Endoscopic retrieval by fragmentation is successful for initial and small gastric bezoars. Pharmacotherapy is inconsistent and some may respond to SSRIs (Selective Serotonin Reuptake Inhibitors). Behavioral modification and psychotherapy are effective and recommended in all patients to prevent relapses. Complications postoperatively include wound infection, perforation, paralytic ileus, fecal leakage from laparotomy wound [11]. Diagnosis and surgical intervention at right time prevents bowel and mesenteric ischemia and gangrene.

\section{Conclusion}

Trichobezoars are rare, benign, surgically treatable condition. It is an important differential diagnosis to be remembered in cases of chronic abdominal pain and epigastric mass. Early diagnosis helps in preventing complications. Recurrence is uncommon. Parental counseling and psychotherapy should be mandatory in follow-up which help in preventing relapses. Most cases are stable and have a favorable outcome. With high level of suspicion, prompt diagnosis and treatment, trichobezoars have a good prognosis. 


\section{References}

1. Mariotto A, Peretti M, Scire G, Mantovani A, Zambaldo S, et al. (2014) Trichobezoars in children: therapeutic complications. La Pediatria medica e chirurgica: Medical and surgical pediatrics. 36(5-6): 101

2. Michele Van Hal SS, Karolina Mlynek, Janet M Kinnane (2017) A bizarre case of jejunal trichobezoar in a 3-year-old boy: case report. Practical Dermatology.

3. Aoi S, Kimura K, Tsuda T (2015) Double and synchronous trichobezoars causing small-bowel obstruction and detected by multidetector computed tomography: report of two cases. Surgery today 45(5): 634637

4. Sanjeev Suman PK, Babita GN Singh (2013) Trichobezoar. Journal of Evolution of Medical and Dental Sciences 2(47): 9195-9200.

5. Chogle A, Bonilla S, Browne M, Madonna MB, Parsons W, et al. (2010) Rapunzel syndrome: a rare cause of biliary obstruction. J Pediatr Gastroenterol Nutr 51(4): 522-523.

This work is licensed under Creative

Commons

DOI: 10.19080/JOJCS.2019.10.555786
6. Hamidi H, Muhammadi M, Saberi B, Sarwari MA (2016) A rare clinic entity: Huge trichobezoar. Int J Surg Case Rep 28: 127-130.

7. Baheti AD, Otjen JP, Phillips GS (2017) A hairy situation: trichobezoar presenting with intussusception, and intestinal and biliary perforation in a child. Radiology case reports 12(1): 42-44.

8. Cho KY, Shim DH, Yoo KT (2014) Small bowel volvulus with a jejunal trichobezoar. Indian Pediatr 51(7): 575-576.

9. Jain M, Solanki SL, Bhatnagar A, Jain PK (2011) An unusual case report of rapunzel syndrome trichobezoar in a 3-year-old boy. Int J Trichology 3(2): 102-104.

10. Charan SPG AV, Kalavant AB, Simha PV and Halgeri AB (2018) Jejunal Trichobezoar-A Rare Cause of Intestinal Obstruction in Children: A Case Report. SM J Pediatr Surg 4(3): 1069.

11. Gorter RR, Kneepkens CM, Mattens EC, Aronson DC, Heij HA (2010) Management of trichobezoar: case report and literature review. Pediatr Surg Int 26(5): 457-463.

Your next submission with Juniper Publishers
will reach you the below assets
- Quality Editorial service
- Swift Peer Review
- Reprints availability
- E-prints Service
- Manuscript Podcast for convenient understanding
- Global attainment for your research
- Manuscript accessibility in different formats
( Pdf, E-pub, Full Text, Audio)
- Unceasing customer service
Track the below URL for one-step submission
https://juniperpublishers.com/online-submission.php

